\title{
Compliance in the Duration of Administration of Anticancer Chemotherapy: Comparative Study of Two Different Administration Modalities (PVC versus IVAD)
}

\author{
Bambara $\mathrm{AT}^{* 1}$, Touré $\mathrm{M}^{2}$, Traoré DTA ${ }^{1}$, Kouassi KKY ${ }^{2}$, Kimso $\mathrm{O}^{2}$ and Traoré $\mathrm{SS}^{1}$ \\ ${ }^{1}$ Cancer Unit of Yalgado Ouédraogo University Hospital, Ouagadougou, Burkina Faso, Africa \\ ${ }^{2}$ Oncology Department of Treichville University Hospital, Abidjan, Africa
}

*Corresponding author: Bambara AT, Cancer Unit of Yalgado Ouédraogo University Hospital, Ouagadougou, Burkina Faso, Africa, Tel: 0022670300070, E-mail: tozoula@yahoo.fr

Citation: Bambara AT, Touré M, Traoré DTA, Kouassi KKY, Kimso O, et al. (2017) Compliance in the Duration of Administration of Anticancer Chemotherapy: Comparative Study of Two Different Administration Modalities (PVC versus IVAD). J Cancer Sci Clin Oncol 4(1): 105. doi: 10.15744/2394-6520.4.105

Received Date: July 15, 2017 Accepted Date: August 25, 2017 Published Date: July 28, 2017

\begin{abstract}
Objective: To evaluate the compliance of chemotherapy administration duration by comparing the peripheral venous catheter (PVC) route with the implantable venous access device (IVAD).

Methods: This was a retrospective study that analyzed 566 cycles of chemotherapy administered by PVC at the Cancer Unit of Yalgado Ouédraogo University Hospital and 258 cycles administered by IVAD at the Oncology Department of Treichville University Hospital in Abidjan. We compared the differences between the programmed duration of chemotherapy and the actual duration of administration according to the two routes of administration. The protocols were grouped into 5 categories (1-5) according to the programmed duration of each cycle ( $<3$ hours, 3-6 hours, 7-12 hours, 12-24 hours, $>24$ hours).

Results: In the PVC group, the absolute average time difference for each group category ( 1 to 5 respectively) was of $42,78,162,93$ and 699 minutes. In the IVAD group, this absolute average time difference was of 4, 15, 10, 74 and 47 minutes for categories 1 to 5 respectively. In the PVC group, chemotherapy sessions were significantly longer than expected and the gap was larger for long session chemotherapies $(\mathrm{p}=0.0001)$. In the IVAD group, chemotherapy was on average significantly shorter than expected and the gap was larger for long session chemotherapies $(\mathrm{p}=0.01)$.

Conclusion: Adherence to nursing good chemotherapy practice recommendations may allow for better compliance of administrative protocols.
\end{abstract}

Keywords: Chemotherapy; Gravity perfusion; Peripheral venous; Implantable venous access device

List of Abbreviations: IVAD: Implantable Venous Access Device; PVC: Peripheral Venous Catheter

\section{Introduction}

Anti-cancer chemotherapy is one of the main therapeutic means against cancer. It is most often done by intravenous infusions of veinotoxic drugs at very regular intervals over several months. This requires good, reliable and comfortable venous access. Implantable central venous access devices, marketed for the first time in 1982, have become indispensable in the administration of chemotherapy [1]. The peripheral venous pathway is used only for short-term infusions of certain drugs, for a reduced number of cures, in patients with a good venous capital [2]. In our resource-limited countries and without a universal health coverage system, implantable central venous catheters are not accessible to all patients. Chemotherapy is given mainly through peripheral venous access, by gravity perfusion and without a flow regulator. The disadvantages of using this route for chemotherapy may compromise the respect of the duration of administration and consequently the efficacy and safety of treatment [3]. We repeatedly analyzed the duration of administration of peripheral venous chemotherapy sessions by comparing them with those administered by implantable chamber catheters. This study could serve as an advocacy tool to facilitate the accessibility of implantable chamber catheters for patients treated with chemotherapy.

\section{Patients and Methods}

This was a retrospective study that analyzed 566 cycles of chemotherapy administered by PVC at the cancer unit at the Yalgado 
Ouédraogo University Hospital and 258 cycles administered by IVAD in the oncology department of the Treichville University Hospital. These chemotherapies involved 137 patients with PVC and 43 patients with IVAD. The cycles were administered in an outpatient hospital by a team of dedicated nurses on a daily rotation basis. The different steps of administration are recorded on an administration sheet, indicating the start and end times of each injection, the flow rate of each infusion (estimated in number of drops per minute).

In the PVC group, cycles were administered by avoiding the wrist and elbow joints. The infusion technique was gravity infusion without a flow regulator. The equipment consisted essentially of an intra-venous infusion set comprising a perforator, a drip chamber, an air vent, a roller clamp, a tube, a Y injection site, and a Luer Lock connection system terminal [4]. The venous approach was performed with a peripheral venous catheter. In the IVAD group, gravity infusion without flow regulator was also used. The equipment also had a three-way valve connected to the tube. The approach to the implantable chamber was made using a Huber* needle.

The beginning of the cycle was considered when the venous route was taken and the removal of this pathway was retained as the end of the cycle. The nursing team adjusts the infusion rate using the roller clamp by counting the number of drops in the dropper chamber. Universal calibration was used, equivalent to 20 drops per milliliter of solution.

Chemotherapy protocols ranged from short-term monotherapies to long-term combinations drugs lasting more than 24 hours requiring hospitalization. Medication doses, volumes of solution, and recommended administration times were clearly indicated on the protocol sheet for the nurse. Each cycle consisted of three main stages: premedication which lasted on average 30 minutes, infusions of anticancer drugs intercalated with short rinses and a final rinse time. The cisplatin-based protocols also included a pre-hydration phase (before premedication) and a post-hydration phase that closed the cycle.

We included all cycles with a completed form including the accuracy of the beginning time of the first and last nursing act related to the administration of chemotherapy. The interrupted cycles for acute toxicity occurring during infusions were not retained. For each cycle, we analyzed the protocol record and calculated the difference in minutes between the expected time and the actual duration time of the administration. The protocols were grouped into 5 categories according to the expected duration of the cycle. Category 1: cycles of less than 3 hours; Category 2: cycles of 3 to 6 hours; Category 3: cycles longer than 6 hours and less than or equal to 12 hours; Category 4: cycles longer than 12 hours and less than or equal to 24 hours; Category 5: cycles of more than 24 hours.

For each category, the expected times were compared to actual duration time by the two groups. Given the heterogeneity of the chemotherapy protocols in each category, we have chosen as the important parameter for each cycle the difference between the expected time and the actual time of administration. These deviations were described by absolute mean difference, mean difference, median difference and standard deviation. The mean differences were compared between the two groups by Student's t-test or the Mann-Withney/Wilcoxon test for categories below 30. A significance level of 0.05 was used for these analyses.

\section{Results}

We analyzed 556 cycles in the PVC group and 258 cycles in the IVAD group for 137 and 34 patients respectively. The expected cycle times ranged from 90 minutes for docetaxel or doxorubicin monotherapies to 56 hours for continuous infusion of 5 fluorouracil chemotherapies. The absolute mean difference for each protocol is represented in Table 1 and 2 . Category 2 cycles

\begin{tabular}{|c|c|c|c|c|}
\hline Categories & $\begin{array}{c}\text { Expected } \\
\text { durations } \\
(\mathrm{mn})\end{array}$ & Protocols & Numbers & $\begin{array}{l}\text { Absolute mean* } \\
\text { differences (mn) }\end{array}$ \\
\hline \multirow{5}{*}{$\begin{array}{c}\text { Catégory } 1 \\
(n=119)\end{array}$} & \multirow{5}{*}[90-180]{} & Docetaxel & 59 & 29 \\
\hline & & $\mathrm{AC}$ & 24 & 55 \\
\hline & & Oxaliplatin & 19 & 37 \\
\hline & & Doxorubicin & 12 & 35 \\
\hline & & Dacarbazin & 5 & 88 \\
\hline \multirow{4}{*}{$\begin{array}{c}\text { Category } 2 \\
(n=276)\end{array}$} & \multirow{4}{*}[180-360]{} & FAC & 217 & 84 \\
\hline & & Carboplatin+Paclitaxel & 43 & 57 \\
\hline & & CHOP & 11 & 66 \\
\hline & & ABVD & 5 & 14 \\
\hline \multirow{2}{*}{$\begin{array}{c}\text { Category } 3 \\
(n=36)\end{array}$} & \multirow{2}{*}[360-720]{} & Cisplatin & 22 & 117 \\
\hline & & ECX & 14 & 231 \\
\hline $\begin{array}{c}\text { (Category 4) } \\
(n=76)\end{array}$ & [720-1440] & CAP & 76 & 94 \\
\hline
\end{tabular}




\begin{tabular}{|c|c|c|c|c|}
\hline Categories & $\begin{array}{c}\text { Expected } \\
\text { durations } \\
(\mathbf{m n})\end{array}$ & Protocols & Numbers & $\begin{array}{c}\text { Absolute mean* } \\
\text { differences (mn) }\end{array}$ \\
\hline \multirow{2}{*}{$\begin{array}{c}\text { Category 5 } \\
(\mathbf{n = 5 9 )}\end{array}$} & {$[1440-3360]$} & Cisplatin+LV5FU & 30 & 1042 \\
\cline { 3 - 5 } & & LV5FU & 16 & 149 \\
\cline { 3 - 5 } & Cisplatin+5Fluorouracil & 13 & 1053 \\
\hline
\end{tabular}

AC: Adriblastin+Cyclophosphamid; FAC: 5Fluorouracil+Adriblastin+Cyclophosphamid; CHOP: Cyclophosphamid+Ad riblastin+Vincristin+Prednisone; ABVD: Adriblastin+Bleomycin+Vincristin+Dacarbazin; ECX: Epirubicin+Cisplatin+

Capecitabin; CAP: Cyclophosphamid+Adriblastin+Cisplatin; LV5FU: Leucovorin+5Fluorouracil

*: Absolute means differences between expected duration and actual duration

Table 1: Absolute mean differences between expected duration and actual duration according to the protocols (PVC group)

\begin{tabular}{|c|c|c|c|c|}
\hline Categories & $\begin{array}{c}\text { Expected } \\
\text { durations } \\
\quad(\mathrm{mn})\end{array}$ & Protocols & Numbers & $\begin{array}{l}\text { Absolute mean* } \\
\text { differences }(\mathrm{mn})\end{array}$ \\
\hline $\begin{array}{c}\text { Catégory } 1 \\
(n=56)\end{array}$ & [90-180] & Docetaxel & 56 & 4 \\
\hline \multirow{2}{*}{$\begin{array}{c}\text { Category } 2 \\
(n=42)\end{array}$} & \multirow{2}{*}[180-360]{} & Carboplatin+Paclitaxel & 24 & 14 \\
\hline & & Paclitaxel+Trastuzumab & 18 & 15 \\
\hline $\begin{array}{c}\text { Category } 3 \\
(n=56)\end{array}$ & {$[360-720]$} & FEC & 56 & 7 \\
\hline \multirow{2}{*}{$\begin{array}{c}\text { Category } 4 \\
\quad(n=28)\end{array}$} & \multirow{2}{*}{ [720-1440] } & Cisplatin + Doxorubicin & 12 & 11 \\
\hline & & Cisplatin+Gemcitabin & 16 & 46 \\
\hline \multirow{4}{*}{$\begin{array}{c}\text { Category } 5 \\
(n=76)\end{array}$} & \multirow{4}{*}[1440-3360]{} & Irinotecan+LV5FU & 18 & 76 \\
\hline & & Folfox 4 & 30 & 9 \\
\hline & & Folfox $4+$ Bevacizumab & 16 & 29 \\
\hline & & TCF & 12 & 124 \\
\hline
\end{tabular}

FEC: 5Fluorouracil+Epirubicin+Cyclophosphamid; LV5FU: Leucovorin+5Fluorouracil; Folfox: LV5FU+Oxaliplatin;

TCF: Dcocetaxel+Carboplatin+5Fluorouracil

*: Absolute means differences between expected duration and actual duration

Table 2: Absolute mean differences between expected duration and actual duration according to the protocols (IVAD group)

( 3 to 6 hours) were the most represented in the PVC group (48.8\%) while in the IVAD group the cycles were mostly category 5 (over 24 hours), that is $29.5 \%$ (Table 1 ). The duration of administration was more respected in the IVAD group than in the PVC group with gaps between the actual duration and the expected duration more often nil; The median difference was 0 minutes for all categories in this group (Figure 1). In the PVC group, the mean difference was $+16,+64,+74,+52,+654$ minutes for categories 1, 2, 3, 4 and 5 respectively. These differences were statistically significant $(\mathrm{p}<0.0001)$. In the IVAD group, these differences were $+0.5,+10,-3,-4$ and -34 minutes for categories $1,2,3,4$ and 5 respectively $(p=0.01$ ) (Table 3 ). Absolute mean difference was significantly higher in the PVC group than in the IVAD group in comparison by category (Table 4).

\begin{tabular}{|c|c|c|c|c|c|c|}
\hline \multirow[b]{2}{*}{ Categories } & \multicolumn{5}{|c|}{ Difference between expected duration and actual duration (in min) } & \multirow[t]{2}{*}{ P value $^{*}$} \\
\hline & Average difference & $\begin{array}{l}\text { Absolute mean } \\
\text { difference }\end{array}$ & Median difference & $\begin{array}{l}\text { Standard } \\
\text { deviation }\end{array}$ & Range & \\
\hline & \multicolumn{5}{|c|}{ PVC Group } & \\
\hline Category 1 & +16 & 42 & 5 & 55,6 & {$[-90 ; 219]$} & \multirow{5}{*}{$<0,0001$} \\
\hline Category 2 & +64 & 78 & 55 & 80,2 & {$[-160 ; 384]$} & \\
\hline Category 3 & +74 & 162 & 40 & 210 & {$[-213 ; 620]$} & \\
\hline Category 4 & +52 & 93 & 40 & 115,1 & {$[-184 ; 370]$} & \\
\hline \multirow[t]{2}{*}{ Category 5} & +654 & 699 & 970 & 790,4 & {$[-703 ; 1660]$} & \\
\hline & \multicolumn{5}{|c|}{ IVAD group } & \\
\hline Category 1 & $+0,5$ & 4 & 0 & 7,4 & {$[-30 ; 20]$} & \multirow{5}{*}{0,01} \\
\hline Category 2 & +10 & 15 & 0 & 27,9 & {$[-62 ; 140]$} & \\
\hline Category 3 & -3 & 10 & 0 & 18,6 & {$[-100 ; 20]$} & \\
\hline Category 4 & -4 & 74 & 0 & 137,1 & {$[-440 ; 310]$} & \\
\hline Category 5 & -34 & 47 & 0 & 99,5 & {$[-380 ; 180]$} & \\
\hline
\end{tabular}

* Test comparing the mean differences (Student's t-test)

Table 3: Means, absolute means, medians, and standard deviations of differences between expected duration and actual duration according to the categories. Intragroup comparisons 


\begin{tabular}{|c|c|c|c|c|c|}
\hline \multirow{2}{*}{ Categories } & \multicolumn{2}{|c|}{ PVC Group } & \multicolumn{2}{c|}{ IVAD Group } & \multirow{2}{*}{ P value $^{*}$} \\
\cline { 2 - 5 } & $\begin{array}{c}\text { Absolute mean } \\
\text { difference }\end{array}$ & $\begin{array}{c}\text { Standard } \\
\text { deviation }\end{array}$ & $\begin{array}{c}\text { Absolute mean } \\
\text { difference }\end{array}$ & $\begin{array}{c}\text { Standard } \\
\text { deviation }\end{array}$ & \\
\hline Category 1 & 42 & 40,1 & 4 & 6,2 & $<0,0001$ \\
\hline Category 2 & 78 & 66,8 & 15 & 25,6 & $<0,0001$ \\
\hline Category 3 & 162 & 151,6 & 10 & 40,5 & $<0,0001$ \\
\hline Category 4 & 93 & 85,3 & 74 & 114,7 & $0,007^{* *}$ \\
\hline Category 5 & 699 & 535,6 & 47 & 93,7 & $<0,0001$ \\
\hline \multirow{2}{*}{ * Student's t-test; ** Mann-Whitney/Wilcoxon Test } \\
Table 4: Absolute mean difference between the categories. Intergroup comparison
\end{tabular}

The differences between actual and expected perfusion time according to the groups and categories are represented in Figure 1 and 2.

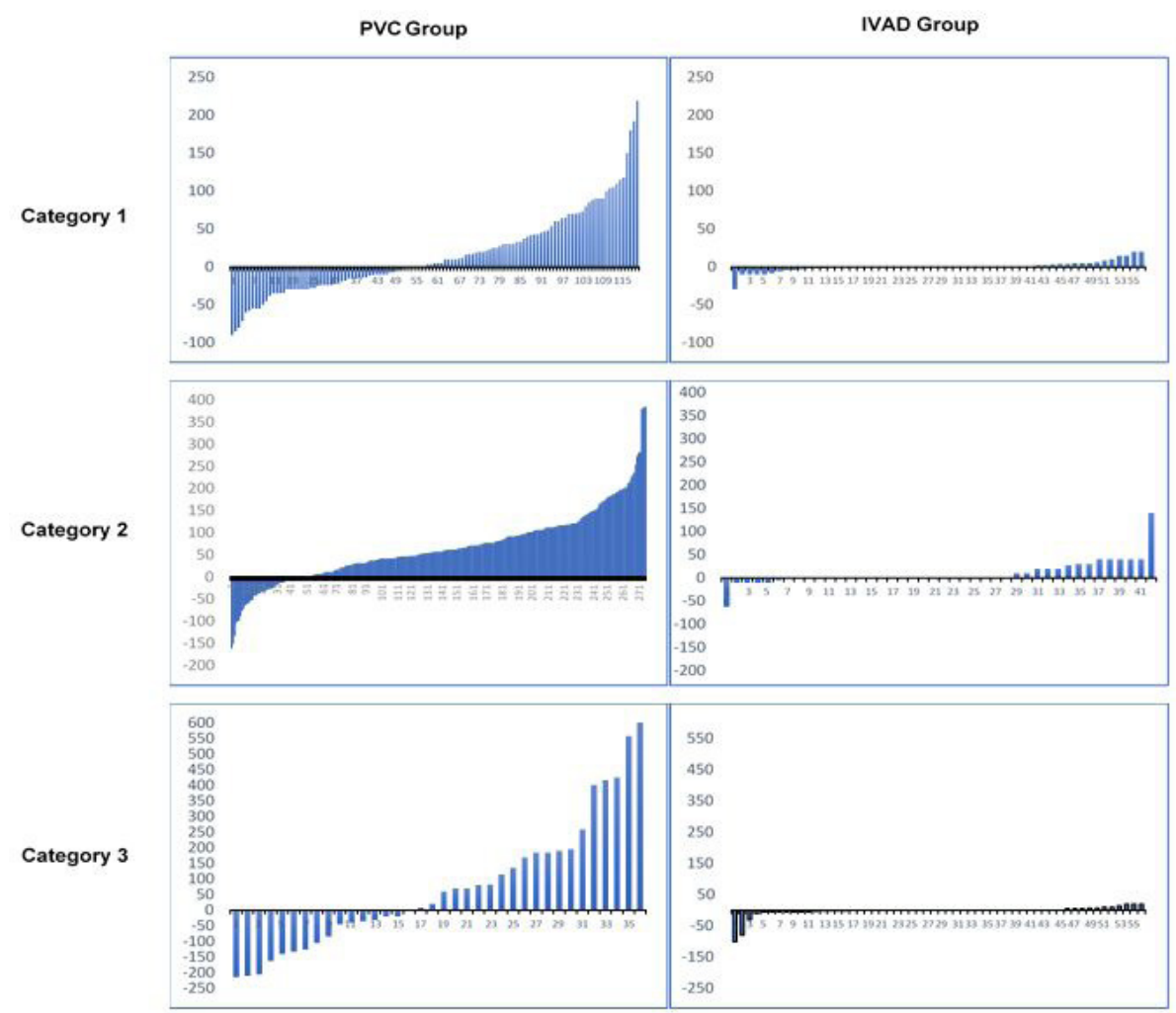

Figure 1: Representation of differences between actual and expected infusion time (in minutes) according to the groups and categories 1,2 and 3
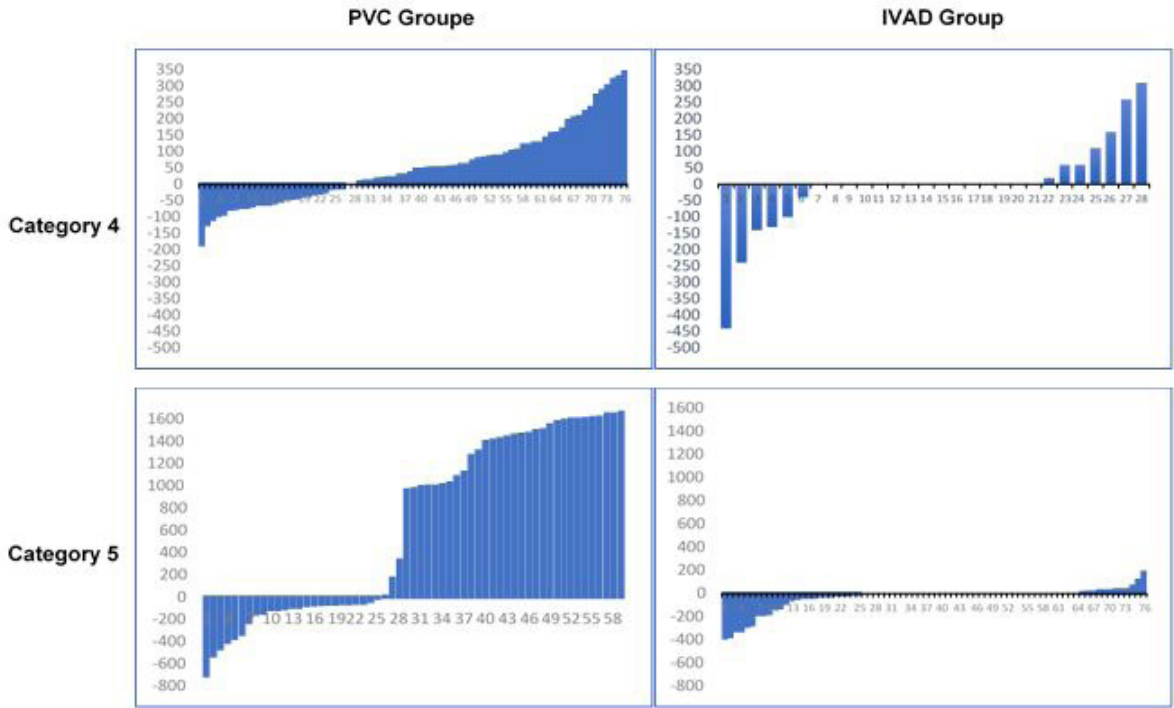

Figure 2: Representation of differences between actual and expected infusion time (in minutes) according to the groups and categories 4 and 5 


\section{Discussion}

Our study revealed significant discrepancies between the programmed time and the actual duration time of administration. These differences were greater in the PVC group than in the IVAD group. Chemotherapies were on average longer than expected in the PVC group while shorter in the IVAD group. Some of the main limitations of our study were its retrospective nature, the heterogeneity of practices from one nurse to another and from one center to another. In addition, the heterogeneity of treatment protocols and infusion setups may have impaired the comparability of administration time from one center to another, from one patient to another and from one cycle to another even for the same patient. Despite these potential sources of bias, this study allowed us to make an inventory of the compliance of chemotherapy administration time.

At the Yalgado Ouédraogo University Hospital in Ouagadougou, chemotherapy is almost exclusively administrated by peripheral venous catheter because of the high cost of implantable chamber catheters and the lack of qualified personnel for the installation. We therefore did not have an IVAD group for an intra-center comparison. The oncology department at Treichville University Hospital, with a cohort of patients treated by central venous system, served as the center for the IVAD group.

Since interrupted chemotherapy cycles for incidents were not included in our study, the non-conformity of the administration time is mainly related to the problems of regulation of infusion rate. Several groups of factors are likely to influence the accuracy of infusion rate. The first group factor relates to the characteristics of the intra-venous infusion set used: internal diameter and material of the tubing, quality of the dropper chamber [5]. The characteristics of the perfusion tubing devices (venous needle size, quality of optional devices), as well as the height of the infusion bag relative to the patient and the length of the infusion line also influence the infusion rate [4]. Some factors are specific to the patient: venous capital, venous pressure, limb with the intravenous set, change of position and movements of the limb with the intra-venous set [6,7]. These patient-related factors are better controlled using an implantable chamber catheter. This could explain the better compliance of the administration time in the IVAD group versus the PVC group and highlights the need to promote the use of the implantable chamber catheter and to ensure its accessibility for patients.

In the PVC group, the differences were greater for long-term chemotherapies. This is due to the accumulation of delays observed for each infusion. The fluorouracil-based protocols over more than 24 hours were administered by gravity infusion due to the unavailability of Elastomeric pump. Patients were kept in hospital and continuous infusion rates were set by the nurses. These low-flow infusions were more exposed to obstructions favored by changes in patient position [8].

In the IVAD group, infusion rates were less exposed to patient-related changes. Indeed, the quality of the venous approach favored by the implantable chamber catheter prevented the abnormal slowdowns in flow rate. On the contrary, flow rates were more likely to accelerate, explaining the shorter-than-expected periods of administration.

We did not find any study that evaluated the duration of administration of peripheral venous catheters during chemotherapy to compare our results with. In general, our study agrees with that of Mayank Dhamija, who showed that errors in administration time were three times more frequent for chemotherapy which lasted more than 1 hour (17\% versus 5\%) [9].

In view of the many factors that can influence the flow of an infusion and the need to ensure a regular flow according to the prescription, flow control devices have been developed since the 1980s. These devices were not used in our study. There is some controversy about their usefulness. In fact, these devices impose compliance with good practice recommendations relating to the height of the perfusion bag and the viscosity of its content, without exempting conventional monitoring of the perfusion rate [10]. Their level of accuracy is therefore a function of whether the manufacturer makes good practice recommendations. Misuse is frequent and potentially dangerous for patients [11].

The administration of chemotherapy is a nursing skill on medical prescription. In addition to factors related to gravity infusion equipment and patient-related factors, the non-compliance with the duration of administration observed in our study could be largely related to nursing practices. Indeed, the absence of a reference system for the administration of chemotherapy explains the heterogeneity of nursing practices which are sometimes harmful. The order of passage of cytotoxic drugs, infusion times may vary from one nurse to another $[10,11]$. These risks are minimized in our study due to the provision of a personalized and nominative administration sheet for each patient and for each cycle. These records indicate the chronological order of administration of each cytotoxic agent as well as the different infusion rates (estimated in drops per minute). The core problem would thus lie in the monitoring of infusions with the regulation of the flow rate by counting drops. Drop counting per minute, which is the only reliable method of controlling flow, is not routinely done. The monitoring of this flow rate throughout the infusion is also not systematic. An audit on gravity infusion is necessary to improve nursing and medical practices to ensure the quality of chemotherapy administration and patient safety.

\section{Conclusion}

This study revealed a very high deviation in the duration of administration which did not comply with the medical prescription. This non-compliance may compromise the efficacy and safety of chemotherapy. Non-compliance of good nursing practices may be the main cause. An audit of the gravity perfusion will provide recommendations for good chemotherapy administration practices. 


\section{References}

1. Gow KW, Tapper D, Hickman RO (2017) Between the lines: The 50 th anniversary of long-term central venous catheters. Am J Surg 213: 837-48.

2. Gallieni M, Pittiruti M, Biffi R (2008) Vascular access in oncology patients. CA Cancer J Clin 58: 323-46.

3. Lévi F (2006) Chronotherapeutics: the relevance of timing in cancer therapy. Cancer Causes Control 17: 611-21.

4. Peterfreund RA, Philip JH (2013) Critical parameters in drug delivery by intravenous infusion. Expert Opin Drug Deliv 10: 1095-108.

5. Flack FC, Whyte TD (1974) Behaviour of standard gravity-fed administration sets used for intravenous infusion. Br Med J 3: 439-43.

6. Carleton BC, Cipolle RJ, Larson SD, Canafax DM (1991) Method for evaluating drip-rate accuracy of intravenous flow-regulating devices. Am J Hosp Pharm 48: 2422-6.

7. Helm RE, Klausner JD, Klemperer JD, Flint LM, Huang E (2015) Accepted but unacceptable: peripheral IV catheter failure. J Infus Nurs 38: 189-203.

8. Wallis MC, McGrail M, Webster J, Marsh N, Gowardman J, et al. (2014) Risk factors for peripheral intravenous catheter failure: a multivariate analysis of data from a randomized controlled trial. Infect Control Hosp Epidemiol 35: 63-8.

9. Dhamija M, Kapoor G, Juneja A (2014) Infusional chemotherapy and medication errors in a tertiary care pediatric cancer unit in a resource-limited setting. J Pediatr Hematol Oncol 36: e412-5.

10. Caruba T, Havard L, Gillaizeau F, Guérot E, Prognon P, et al. (2009) Evaluation of flow rate regulators for intravenous infusion. Ann Fr Anesth Reanim 28: 936-42.

11. Le Reste C, Fiedler A, Dubois S, Dewailly A, Le Du I, et al. (2015) How to promote the respect of good infusion practices by meeting health care professionals? Ann Pharm Fr 74: 232-43. 